“siRNA and RNAi optimization” Alagia, A., Eritja, R. WIREs RNA, in press (2016).

doi: 10.1002/wrna.1337

\title{
SiRNA and RNAi Optimization
}

\author{
Adele Alagia and Ramon Eritja
}

Keywords: RNAi, siRNA,miRNA, RISC, Ago2, Off-target effects, chemical modification, structural modification, delivery, exosomes, RNA microsponges, HDL-like particles, dumbbell, si-siRNA, aiRNA, asiRNA, is-siRNA, dsi-RNA, ss-siRNA

\begin{abstract}
.
The discovery and examination of the post-transcriptional gene regulatory mechanism known as RNA interference (RNAi), contributed to the identification of small interfering RNA (siRNA) and the comprehension of its enormous potential for clinical purposes. Theoretically, the ability of specific target gene downregulation makes the RNAi pathway an appealing solution for several diseases. Despite numerous hurdles resulting from the inherent properties of siRNA molecule and proper delivery to the target tissue, more than 50 RNA-based drugs are currently under clinical testing. In this work we analyze the recent literature in the optimization of siRNA molecules. In detail, we focused on describing the most recent advances of siRNA field aimed at optimize siRNA pharmacokinetic properties. Special attention has been given in describing the impact of RNA modifications in the potential off-target effects such as saturation of the RNAi machinery, passenger strand-mediated silencing, immunostimulation and miRNA-like off-target effects as well as to recent developments on the delivery issue. The novel delivery systems and modified siRNA provide significant steps towards the development of reliable siRNA molecules for therapeutic use.
\end{abstract}

\section{Introduction}

The breakthrough of RNAi phenomenon ${ }^{1,2}$ has incited the discovery of the core proteins of RNA-induced silencing complex (RISC) ${ }^{3,4}$, the identification of the silencing trigger molecule ${ }^{5}$ and the design of synthetic siRNA duplex ${ }^{6}$. Later on, the thrilling idea of siRNA target-specific signature, due to the necessity of perfect identity with its cognate messenger RNA (mRNA), had to deal with the silencing of unintended targets ${ }^{7}$. Furthermore, because of the inherent siRNA limitations (poor bioavailability, instability against nucleases, hydrophilicity and polyanionic nature), the development of RNAi-based therapeutics had suffered a serious slowdown. However, the ease of customization, the possibility of treating "undruggable" diseases and shortening the drug discovery process, have made siRNA-based therapeutics appealing agents for future tailored medicine (Figure 1). 


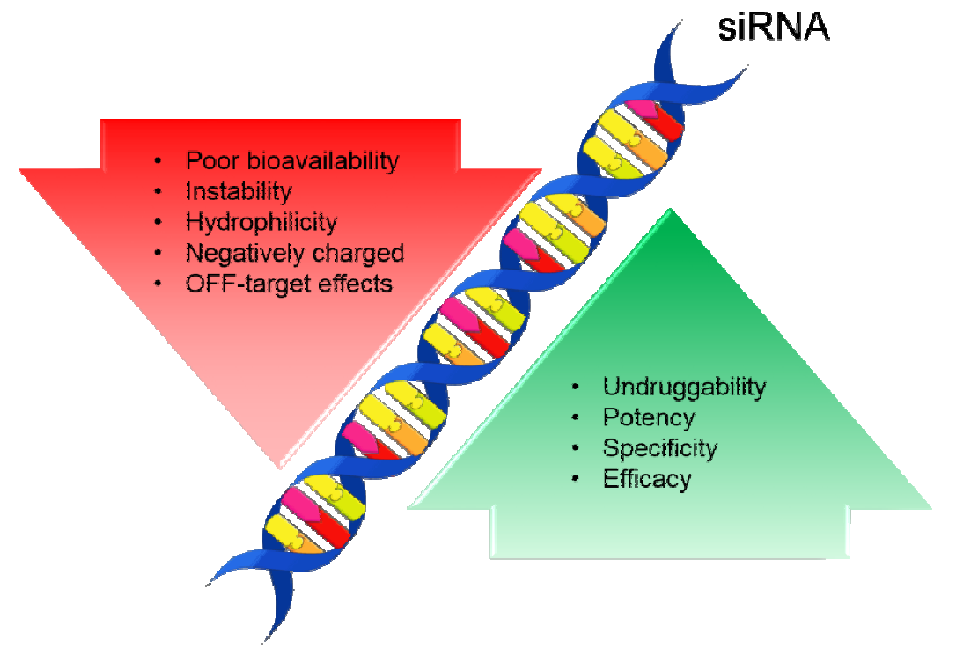

Figure 1. Benefits and drawbacks of siRNA-based therapeutics. In addition to important unwanted off-target effects such as the up- and down-regulation of thousands of unrelated genes, during the attempt of develop RNAi-based therapeutics some intrinsic siRNA limitations have been identified. Upon entering the bloodstream the siRNA molecules are promptly degraded and eliminated, the action of ribonucleases and reticuloendothelial system (RES) compromises its bioavailability under physiological conditions. Furthermore, the siRNA hydrophilicity, high molecular weight and polyanionic nature restricts its passive diffusion across cellular membranes. Even though all these serious issues, the siRNA properties can be adjusted in terms of gene silencing abilities (potency, specificity and efficacy). The ability of siRNA molecule to target any disease-related gene offers a valuable option to block the production of all those undruggable proteins, not-responding to traditional therapy, at the messenger RNA level.

Theoretically, the siRNA nucleotide sequence should determine the specificity, potency and efficacy of siRNA-mediated gene silencing, but many factors influence its reliability. For example, the specificity, the ability to knockdown the target gene without interfering with the expression of other genes, is basically compromised by partial complementarity between the siRNA sequence and unintended targets. To ensure exact recognition of the target mRNA, chemical/structural modifications supported by bioinformatics tools have been extensively employed $^{8,9}$. But despite the great deal of efforts, the specificity remains one of the most challenging tasks for siRNA optimization. In addition, the mRNA accessibility affects the siRNA efficacy. The presence of secondary/tertiary structures on mRNA transcripts hinders the hybridization between the siRNA-programmed RISC and the target site sequence ${ }^{10,11}$. Since excess amounts of siRNA saturate the RNAi machinery, the design of powerful siRNA molecules is indispensable. More powerful siRNA duplexes have been successfully synthesized using several chemical modifications. ${ }^{12}$. Finally, the implementation of siRNA activity for clinical applications should be addressed by the design of carriers able to deliver the silencing cargo into specific tissue. To enhance the siRNA bioavailability, its stability into biological milieu and overcome the physiological barriers after systemic administration, many delivery strategies have been evaluated ${ }^{13}$. Hence, the achievement of an optimized siRNA design strategy is possible, only taking into account all these issues. Here, we presented an overview focusing on the 
recent progress in siRNA field aimed at optimize siRNA pharmacokinetic properties. Furthermore, we outlined the recent advancements in delivery strategies.

\section{Dissecting the siRNA: chemical modifications and siRNA structure.}

\subsection{How Argonaute splits the siRNA molecule}

Argonaute proteins are the fundamental components of the small-RNA-driven gene silencing mechanism. In human, eight Argonaute proteins have been identified; four of them belonging to Argonaute subcategory (hAgo 1-4) and the other four to the PIWI subcategory (hPIWI 1-4). Among them, only Ago2 possesses the ability to cleave the target $\mathrm{mRNA}^{14}$. Following the discovery of the core protein of RNAi pathway, intensive studies have defined its domain composition. The hAgo2 has revealed cradle shaped architecture and highly conserved domains: MID, PIWI, PAZ and N-lobe (Figure 2). The MID interacts with the first nucleotide (5'-

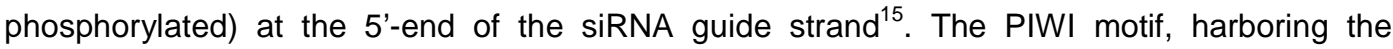
RNase-H like catalytic core of Ago2, is responsible for the endonucleolytic cleavage of the target $m R N A^{16}$. The PAZ domain is a hydrophobic cavity able to recognize the $3^{\prime}$ terminal dinucleotide overhang of the siRNA strand ${ }^{17}$. Finally, the N-lobe acting as a molecular wedge, is strictly required for duplex unwinding and maturation of $\mathrm{RISC}^{18}$. Thanks to the $3^{\prime}$ terminal unpaired dinucleotide structures and 5' phosphate group, the Argonautes can efficiently identify the siRNA/miRNA (microRNA) molecules among other small RNAs ${ }^{19}$. Moreover, the RISC is able to discriminate between double stranded (ds) RNA and dsDNA molecules, only the formers are powerful triggers of gene silencing. Unlike dsDNA, that adopts a B-form conformation (righthanded helix, 10bp per helical turn and $20 \AA$ in diameter), dsRNA displays an A-helix motif and one helical turn containing $11 \mathrm{bp}$ with a diameter of $23 \AA$. The wider major groove of the A-form helix is needed for stable interactions with RISC and target $\mathrm{mRNA}^{20}$.

Upon RISC assembly and the degradation of the passenger strand, Ago2 functionally classifies the guide strand into 5 discrete sections: (i) anchor; (ii) seed; (iii) central; (iv) 3' supplementary and (v) tail ${ }^{21}$ (Figure 2). 


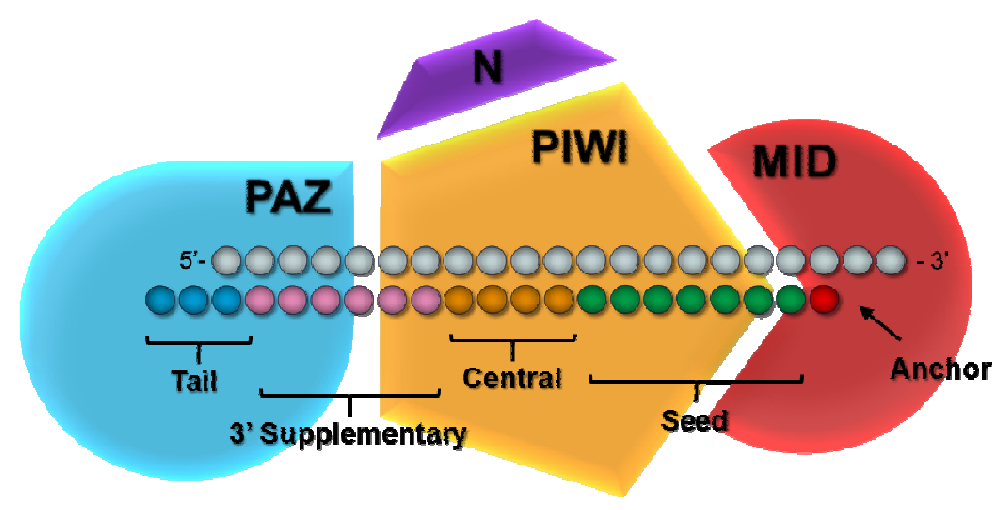

Figure 2. Ago2 diagram and siRNA molecule. Top strand is the passenger or sense, bottom strand is the guide or antisense. The passenger strand during RISC maturation is nicked and degraded, whereas the guide strand is retained and serves as complementary sequence for the identification of the target mRNA.

\subsubsection{The anchor site (1)}

The first nucleotide of the guide strand (Figure 2 in red) is stably buried into the MID domain of Ago2 protein and is not actively engaged in target recognition ${ }^{22}$. Moreover, the presence of phosphate group at 5' terminus is essential for siRNA efficacy ${ }^{23,24}$, the phosphorylation of the 5'end is also required for strand loading and proper Ago2-mediated cleavage.

Even though the MID pocket provides adequate space to the accommodation of the natural bases, the presence of uridine or adenine is preferred over guanine and cytosine ${ }^{15,25}$. The interactions between the MID cleft and the different natural bases are essentially not-specific and the identity of the interacting MID-nucleotide does not disturb the overall protein structure. Detailed studies on nucleobase recognition by the MID domain have revealed the presence of a well-arranged water network able to mediate contacts between several residues (Met 437, Lys 440, Ile 477 and Asp 480) and the N6 of the adenine ${ }^{26}$. Furthermore, Schirle and co-workers have suggested that the identity of the first nucleotide of the guide strand is not decisive for the initial recognition of the target mRNA but is crucial for increasing the stability between the Ago2 and the mRNA after seed-pairing nucleation.

Computational screenings of MID pocket and in vitro tests have also identified new 5 ' end modifiers able to enhance siRNA potency ${ }^{27}$. For example, bulky adenosines analogues have demonstrated to accommodate poorly into the MID pocket, their introduction at the 5'end of siRNA molecules were detrimental for RNAi activity in comparison with the natural adenosine. On the other hand, siRNAs modified with triazole derivatives, fitting better into the 5'-nucleotide binding pocket, have revealed stronger silencing activity with respect to unmodified siRNA molecules. 


\subsubsection{The seed region (2-8)}

The stretch from position 2 to 8 (Figure 2 in green), well known as the seed, is responsible for the initial base-pair formation during the nucleation step of target recognition ${ }^{28}$. Proper seed pairing is important for both miRNA and siRNA target recognition ${ }^{29}$, but is also critical for serious unintended miRNA-like OFF-target effects ${ }^{30}$. SiRNA molecules with different AU or GC content in the seed region have revealed opposite performance in terms of seed-matched target(s) silencing. The presence of four to seven $\mathrm{A} / \mathrm{Us}$ in the seed stretch has proved to effectively reduce the seed-off target silencing while sustaining high activity for target gene ${ }^{31}$. Thus, the reduction of seed thermodynamic stability can diminish the siRNA seed-dependent off-target effects $^{32}$. Moreover, the modification of the seed sequence with UNA (Unlocked Nucleic Acid) moieties has demonstrated to reduce the siRNA off-target potential. The presence of UNA modification locally destabilizing the RNA-RNA hybridization impacts more heavily on partial target recognition, responsible for the miRNA-off target effect, rather than on fully complementary target binding. For instance, the introduction of one UNA unit at 3'-end of the seed stretch has dramatically reduced the silencing mediated by partial complementarity without affecting the siRNA potency ${ }^{33}$.

Even though strong interactions can be prevented using AU-rich seed siRNAs or UNA seed modified siRNA, it was demonstrated that 3 ' supplementary region actively contributes to OFFtarget effects, neutralizing the influence of 5 ' base pairing ${ }^{34,35}$.

The modification of guide seed with 2'-O-methyl-RNA moieties (Figure 3), especially at position 2 of the guide strand, has also resulted in reduced silencing of off-target transcripts ${ }^{36}$. Of note, position 2 of the guide strand also plays an important role in ON-target recognition, 2'-Omethoxyethyl-RNA modification (Figure 3) has demonstrated to be detrimental for adequate target binding and Ago-mediated cleavage ${ }^{23}$. To diminish the stability between guide seed and mRNA, the seed region was also replaced with DNA units ${ }^{37}$. Since the DNA/RNA hybrid is less stable than RNA homoduplex, weaker interactions help to hinder the target duplex nucleation in absence of supplementary interactions beyond the seed sequence. Because the seed portion needs to be arranged within the Ago into a perfectly ordered A-form helix ${ }^{38}$, the DNA/RNA helical deviation from A-form geometry is most likely detrimental for target recognition and seedmediated activity. Further studies, on correlation between helical distortion and proper target binding, remarked upon the importance of A-form duplex geometry for the Ago-mediated mRNA initial recognition. Increasing numbers of mismatches along the seed stretch, entailing local distortion of the duplex structure, lead to a general "decoupling" between the guide and the mRNA. Central seed mismatches ( $4-5$ positions) heavily weigh on Ago processivity more than those closer to the seed ends ${ }^{21}$. Furthermore, single wobble base pair (G:U) within the seed sequence has demonstrated to be a good strategy able to efficiently abrogate the off-target silencing ${ }^{39,40}$. Thus, both duplex stability and structural parameters contribute to the effectiveness of guide-mediated off-target silencing. 

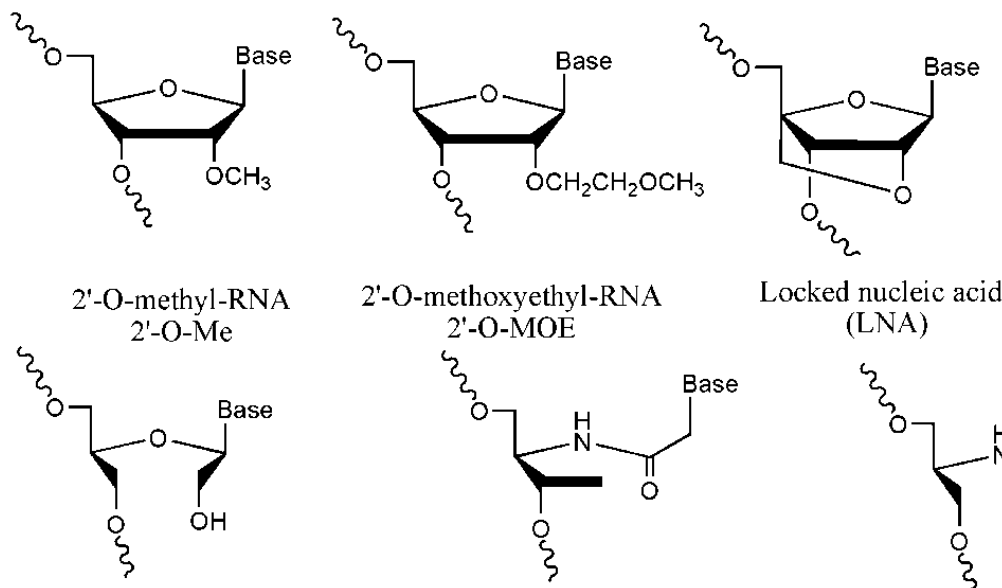

Unlocked nucleic acids (UNA)

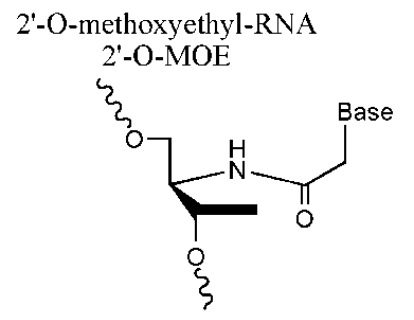

Threoninol nucleic acids (aTNA)

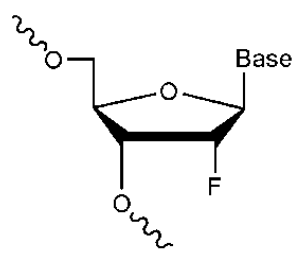

2'-F-RNA

$\left(2^{\prime}-\mathrm{F}\right)$

Figure 3. Chemical structures of modified backbones used in siRNA.

\subsubsection{The central region $(9-12)$}

Central base pairs (nucleotides $9-11$ ) (Figure 2 in yellow) lying within the catalytic part of the Ago2 protein support the cleavage of the target mRNA. Central base pair matches are required for RISC maturation and for the correct conformation of the Ago's active site. Passenger strand is the first RISC target, its cleavage helps the dissociation from the guide strand and the production of the active RISC ${ }^{41}$. Slicer-dependent maturation of the RISC is the main mechanism for the elimination of the passenger strand. Phosphorothioate substitution of the scissile phosphodiester group makes the passenger strand non-cleavable and promotes a slower bypass mechanism in which the duplex unwinding is cleavage-independent ${ }^{42,}{ }^{43}$. The substitution of central nucleotide pairs with DNA units has demonstrated to lower the silence activity, the deviation from canonical A-form structure of the guide:mRNA duplex likely leads to poor cleavage capability. On the other hand, replacement of central nucleotides either on guide or passenger strand marginally affects the inhibition, probably because hybrid DNA/RNA molecules, assuming a helical conformation between the A- and B-form, are less detrimental for the slicing ${ }^{44}$. Modifications, which prefer $\mathrm{C}^{3}$ '-endo conformation, and stabilize the local RNA structure in A-form geometry (i.e. 2'-O-methyl, 2'-Fluoro, 2'-O-methoxyethyl, Pseudouridine, 2'Thiouridine), have proved to be tolerated at central positions of guide $\operatorname{strand}^{40}, 23,45,46$. Conversely, more flexible units such as Dihydrouridine and L-threoninol-thymine (Figure 3), increasing the flexibility of duplex geometry inhibit the silence activity ${ }^{40,47}$. Thus, proper RNA Aform geometry, around the cleavage site, is essential for adequate Ago2-mediated catalysis. The perturbation of Ago2 catalytic efficiency in presence of central mismatches has once more confirmed the importance of proper duplex arrangement within the cleavage site $^{21}$.

\subsubsection{The 3'-supplementary region (13-18)}


The 3' supplementary region (Figure 2 in pink) is responsible for the propagation of duplex formation over the central cleavage region. In binary complex, the nucleotides 14-18 are surrounded by a narrow channel formed between the PAZ and $\mathrm{N}$ domains, their base stacking interactions are broken and nearly all Watson-Crick edges point inward. The extensive contacts with the Ago2 protein prevent the hybridization between the guide 3'-half and the complementary target. Conversely, the arrangement of guide-target pairing beyond the 5'-half portion leads to significant conformational changes, the PAZ-N channel gets wider and the guide 3'-half is repositioned forming A-form helix ${ }^{22}$. Stable hybridization between the stretch 1418 and the target also facilitates the correct positioning of central nucleotides and the cleavage activity $^{28}$.

\subsubsection{The tail region $(19-21)$}

The terminal part of the guide strand spans nucleotides $19-21$ (Figure 2 in blue) and comprises a dinucleotide-unpaired structure, named overhang, which protrudes from the siRNA duplex and specifically interacts with PAZ pocket ${ }^{19}$. Even though the 3 '-overhang does not actively participate to target recognition, its presence is critical for siRNA activity ${ }^{48}$. Along with the interaction between the 5'end and the MID pocket, stable anchoring of 3'-end within the PAZ domain is essential for the proper unwinding and passenger release ${ }^{28,49}$. During the formation of catalytically competent RISC the passenger strand is nicked and discarded, its elimination permits the propagation of base pairing towards the guide 3 '-end and facilitates the displacement of the overhang from the PAZ cleft. Once the cleaved products are released, the PAZ domain again fastens the guide 3'-end. The PAZ motion, according to the "two state" model, has permitted the elucidation of the siRNA-programmed RISC catalysis as a multiple turnover process ${ }^{50}$. The lodging/dislodging motion is regarded to be a decisive factor in boosting siRNA potency. Likely, more affinity for the PAZ cleft, favoring a rapid increase of the fasten/unfasten rate, accelerates the RISC cleavage turnover. So, in view to improve siRNA potency, through the strengthening of the interactions between the 3' guide overhang and the PAZ pocket, numerous overhang modifications (Figure 3) have been tested ${ }^{51-55}$. Of note, the size of overhang modification, affecting the proper accommodation into the PAZ hydrophobic cavity, influences the siRNA activity ${ }^{56-58}$. Because of the extreme RNA instability in biological milieu, overhang modifications have been primarily developed to protect the siRNA integrity from the action of nucleases ${ }^{6}$. Thereafter, the detection of siRNA-associated side effects has led to evaluate the ability of overhang-modified siRNAs in promoting the correct strand selection ${ }^{59}$. For example, thanks to different binding affinity of modified overhang, chemically asymmetric siRNAs have demonstrated to improve the loading of the intended strand ${ }^{60}$. Indeed, the enhanced siRNA potency is likely the result of preferential loading of the strand bearing the overhang with better PAZ binding affinity. The favored guide strand incorporation impedes the passenger strand misloading, preventing the passenger-mediated OFF-target effects. Remarkably, the absence of passenger strand overhang, eliminating the structure recognized by the PAZ domain, promotes the loading of the strand bearing the overhang ${ }^{61}$. Furthermore, 
overhang-modified siRNAs have extensively employed to induce long-term gene silencing. Enhanced stability towards nucleases has been assumed to endure the silencing effects, even if later studies have clarified that the silencing duration in vitro depends mostly on the cellular doubling-time rather than improved stability ${ }^{62,63}$. Thus, nuclease-stabilized siRNA has more influential impact during in vivo delivery of naked molecules ${ }^{64}$.

\section{Handling the OFF-target effects (OTEs)}

The development of siRNA-based therapeutics, straight after an initial burst, has suffered a setback caused by ever-growing issues questioning on their biological safety. Unintended sideeffects of siRNA-mediated gene silencing essentially hinge upon: (i) suppression of non-target genes; (ii) saturation of RNAi machinery; (iii) passenger strand mediated silencing; (iv) immune response activation (Table 1).

\begin{tabular}{|c|c|c|}
\hline OFF-target type & Effects & Solutions \\
\hline $\begin{array}{l}\text { Saturation of RNAi } \\
\text { machinery }\end{array}$ & $\begin{array}{l}\text { Up-regulation of miRNA- } \\
\text { controlled genes }\end{array}$ & $\begin{array}{c}\text { Improve siRNA potency by chemical } \\
\text { modifications }{ }^{12,58} \text {, use highly potent siRNA } \\
\text { molecules }^{59,60}\end{array}$ \\
\hline $\begin{array}{l}\text { Passenger strand- } \\
\text { mediated silencing }\end{array}$ & $\begin{array}{l}\text { Down-regulation of mRNA } \\
\text { target(s) partially and/or } \\
\text { fully complementary to } \\
\text { passenger strand }\end{array}$ & $\begin{array}{l}\text { Enhance thermodynamic asymmetry, } \\
\text { blunt end siRNA }{ }^{53}, \text { si-siRNA }^{67} \text {, asiRNA }{ }^{53,} \\
\text { 68, 69, aiRNA }{ }^{70}, \text { ss-siRNA }^{71} \text {, block } \\
\text { passenger } 5^{\prime} \text {-end phosphorylation }{ }^{63-65} \text {, } \\
\text { dual targeting siRNA }{ }^{73,74,75}\end{array}$ \\
\hline $\begin{array}{l}\text { Seed-mediated } \\
\text { silencing }\end{array}$ & $\begin{array}{l}\text { Down-regulation of } \\
\text { partially complementary } \\
\text { genes }\end{array}$ & $\begin{array}{l}\text { Avoid common seed complementary } \\
\text { sequences }^{98}, \mathrm{AU} \text {-enriched seed }\end{array}$ \\
\hline Immunostimulation & $\begin{array}{l}\text { Induction of inflammatory } \\
\text { cytokines and type-I } \\
\text { Interferons }\end{array}$ & $\begin{array}{l}\text { Avoid immunostimulatory motifs }{ }^{78,79}, \\
\text { escape the immune system recognition by } \\
\text { chemically modified siRNAs }\end{array}$ \\
\hline
\end{tabular}

Table 1. Schematic summary of siRNA-mediated OTEs

\subsection{Saturation}

The estimated RISC concentration inside the cells ranges between $3-5 \mathrm{nM}$. RISC amount, considering the average volume of a mammalian cell $\left(10^{-13} \mathrm{~L}-10^{-12} \mathrm{~L}\right)$, is in the order of about $10^{3}-10^{4}$ molecules ${ }^{65}$. Thus, saturation of the RNAi machinery is theoretically reached with 10 $100 \mathrm{pM}$ siRNA, which corresponds to $10^{3}-10^{4}$ dsRNA molecules. The transfection of exogenous siRNAs principally leads to competition for RISC binding between the endogenous miRNA pool and the exo-siRNAs. MiRNA displacement influences the miRNA-mediated 
silencing and leads to an overall upregulation of miRNA-controlled genes. The preservation of robust silencing effects with lower dose is advantageous in terms of RISC competition. For this reason, the design of potent siRNA molecules fundamentally aims at the prevention of RISC clogging. If only a fraction of cellular RISC pool is actively occupied by the exo-siRNAs, more RISC molecules are available for endogenous pathways.

Besides canonical modified siRNAs (extensively reviewed elsewhere ${ }^{12,66}$ ), which demonstrated to be more potent with respect to the unmodified ones, innovative approaches based on new siRNA structural variants have been applied. For instance, Dicer substrate interfering RNAs (DsiRNAs) have demonstrated stronger activity with respect to canonical 21-nt siRNAs. Dicer executing the conversion of DsiRNA precursor into active siRNA, assists the Ago loading and raises the RISC incorporation rate. In addition, adequate strand polarity conferred by DsiRNAs minimizes the incorporation of the passenger strand into the RISC ${ }^{67}$. Another successful example of potent siRNA precursors emerges from the synthesis of dumbbell-shaped circular siRNA: a circular RNA molecule that folds into a central stem region and 2 terminal loop structures. Interestingly, dumbbell-shaped siRNA, with 23-bp stem region and 9-nt long loops, has disclosed powerful improvement on silencing activity with respect to canonical 21-bp siRNA. The lack of loose ends, distinctive of siRNA structure, entails better resistance towards nucleases and reduced activation of innate immune response. Furthermore, the slow Dicer processing of dumbbell siRNA, likely allowing a gradual but constant production of active siRNA molecules, drives long-lasting silencing ${ }^{68}$.

\subsection{Passenger strand mediated silencing}

The claim assuring:" the strand with less stable 5'-end is preferentially incorporated into RISC and serves as the guide", holds back the possibility of improper strand incorporation. It has been established that passenger strand could be efficiently loaded as the guide strand, provoking the downregulation of complementary target(s $)^{69}$. Passenger strand-driven silencing compromises siRNA specificity and engulfs the RNAi machinery with the wrong strand. To promote the correct strand election, chemical and structural approaches have been investigated. Since the presence of $5^{\prime}$ terminal phosphate is strictly required during RISC assembly and is essential for silencing activity ${ }^{70}$, chemical modifications able to block the Clp-1mediated 5'end phosphorylation, permit desirable strand incorporation or limit passenger strand activity $^{71,35,72,73}$. Moreover, passenger seed substitutions, altering the duplex stability between the modified strand and the target mRNA, prejudice its ability to participate in target cleavage ${ }^{33}$, $35,47,54$. Hence, strand-blocking properties of chemical modifications are successfully exploited to weaken the passenger strand-mediated silencing. It was also noted, that in the absence of passenger overhang, the activity and the strand selection of blunt siRNAs incline towards the guide strand ${ }^{61}$. Given that the presence of overhang is decisive for PAZ-mediated RISC recruitment, the asymmetrical structure of blunt siRNA promotes the loading of the strand carrying the overhang. 
In order to improve the poor inherent siRNA properties (especially the specificity), novel siRNA architectures have been analyzed. For example, new siRNA construct has originated as a result of the development of LNA (Locked Nucleic Acid, Figure 3) modification. Internal segmented siRNA (si-siRNA) consisting of a 22-nt guide strand and 2 segmented $(10+12 \mathrm{nt})$ passenger strands has demonstrated to be as effective as canonical siRNA. Furthermore, the threestranded siRNA composition, combining the LNA stabilization effect on passenger strands and the silencing inability of shorter passenger strand portions, has proven to be an highly specific siRNA architecture ${ }^{74}$. Therefore, passenger shortening can be an intriguing approach to the elimination of passenger strand-mediated silencing. Asymmetric shorter duplex siRNA (asiRNA), asymmetric siRNA (aiRNA) and blunt end siRNA ${ }^{61,75-77}$ have exhibited silencing ability comparable to canonical siRNA structure and less siRNA-mediated off-target effects. Interestingly, the progressive passenger strand trimming from its 5 '-end does not compromise RISC assembly and its off-target effects. On the other hand, the shortening of the passenger 3'end promotes the preferential loading of the opposite strand. Even though the thermodynamic asymmetry of siRNA duplex contributes to guide strand selection, the presence of the 3' overhang substantially weighs on strand preference.

Since active RISC needs only one strand to execute the cleavage of the target mRNA, singlestranded siRNA (ss-siRNA) was able to trigger gene silencing ${ }^{78}$. This strategy completely abolishes the passenger-mediated silencing but has disclosed decreased potency with respect of double stranded siRNAs. Furthermore, 5 ' unphosphorylated ss-siRNA are not functional, only the chemical addition of a phosphate at $5^{\prime}$-end rescues the silencing activity ${ }^{79}$. In singlestranded fashion, the endogenous phosphorylation is likely hindered, so only prephosphorylated ss-siRNA can stably interact with the RISC. An appealing solution to passenger off-target effects came from dual targeting siRNAs, which harbor two functional strands. Since each strand individually recognizes distinct targets, the passenger-driven silencing is virtually

eliminated ${ }^{80,81}$. Fundamentally, the key steps for correct dual-targeting design are the choice of target genes and the calculation of termini stability. Bioinformatics analysis on gene candidates facilitates the selection of partial overlapped sequences able to form stable multi-bulged duplex siRNA. Moreover, computation of balanced ends permits unbiased strand incorporation and confers comparable effectiveness to both strands ${ }^{82}$.

\subsection{Immunostimulation}

The innate immune system has evolved to support rapid response by the recognition of pathogen hallmarks. Pattern recognition receptors (PRRs) identify pathogen-associated molecular patterns (PAMPs) exclusively present on microorganisms. The innate immune sensors perceive exogenous RNAs as a viral infection, the resulting activation of the defense mechanisms leads to the production of type I interferon and pro-inflammatory cytokines. Sensors designated to the recognition of non-self RNAs belong to TLR (Toll-Like Receptor) and non-TLR pathways ${ }^{83}$. TLRs receptors located at endosomal compartment are the first line of 
defense against viral RNA infections. Their almost exclusively compartmentation also dictates the discrimination between host and non-self nucleic acids. TLRs (TLR3, TLR7, TLR8 andTLR9) specifically interact with stimulatory motifs present on double and single-stranded $\mathrm{RNA}^{84}$. Even though it was noted that some secondary structures stimulate the activation of TLRs, their activation is mostly sequence-dependent. Indeed, polyuridine tracts and GU-rich sequences, are strong RNA immunostimulatory motifs that should be avoided in tailoreddesigned siRNA ${ }^{85,86}$. Among the most important no-TLR receptors, PKR, RIG-I and OAS recognize dsRNA in a sequence-independent manner ${ }^{87}$. DsRNA-dependent protein kinase (PKR) and 2'-5'-oligoadenylate synthetase (OAS) activation restricts viral transcription and translation by blocking cellular protein synthesis and induction of apoptosis ${ }^{88}$. The 5'triphosphate presence on dsRNA structures triggers RIG-I-mediated antiviral signaling ${ }^{89}$. Many efforts have been made to develop siRNA molecules able to escape the innate immune recruitment. SiRNA chemical modifications, able to mitigate the activation of immune response, permit to identify the basic hallmarks recognized by the immune sensors. Ribose 2'-position modifications, such as 2'-OMe, 2'-F and 2'-H, have demonstrated to strongly reduce the levels of IFNs while retaining optimal siRNA-silencing ${ }^{90}$. New approaches based on minor-groove base modifications have aimed at protection of immunostimulatory hot spot present on miRNAmimic $^{91}$. Furthermore, adenine substitution over the other nucleotides has beneficial effects on eluding the immune recognition ${ }^{92,}{ }^{93}$. Although the necessity of neutralizing the immunostimulatory potential of siRNA molecules is fundamental goal to achieve for reliable therapeutic purposes, the siRNA adjuvant effect has been exploited to activate the cellular immunity. A promising approach for cancer and viral therapy came from the development of immunostimulatory siRNAs (is-siRNAs) ${ }^{86,94}$. These bifunctional molecules, linking potent gene silencing properties to suitable production of IFNs, can effectively control chronic viral diseases such as HIV-AIDS, HBV and HCV infections ${ }^{95-97}$. Furthermore, the application of this new siRNA design can not only overcome the cancer drug resistance, but also strengthen the immune surveillance usually evaded by cancer cells ${ }^{98-102}$

\section{4 miRNA-like OTEs}

Analysis of multiple microarray datasets has revealed widespread siRNA-driven gene downregulation ${ }^{30,103}$. The mechanism underlying siRNA off-targeting resembles that miRNA-based: seed base-pairing interactions between the guide strand and the mRNA (especially in the 3'UTRs), are sufficient to suppress the expression of unintended targets ${ }^{7}$. MiRNA-like silencing produces false-positive data and toxic phenotypes contributing to the problematic interpretation of silencing outcomes and weakening the potential therapeutic benefits of siRNA-based drugs. To mitigate the off-target effects, several modifications such as single nucleotide bulge, UNA and 2'-OMe moieties were introduced within seed sequence. The presence of these modifications has proven to ameliorate the siRNA specificity without reducing the siRNA efficiency ${ }^{30,35,104}$. To prevent the miRNA-like silencing, an alternative path comes from the study 
of seed complement frequencies (SCFs) in the 3'UTR transcriptome. It is well-described that each siRNA molecule presents specific off-target fingerprint. Fluctuations in off-target signature essentially rely on seed nucleotide sequence mirroring the complementary target abundance in the 3'UTR genome. Thus, fewer off-target silencing and lower false positive scores can be obtained avoiding the introduction within the seed portion of high-frequency seed complements ${ }^{105}$. Since seed-mediated recognition of complementary transcripts is sufficient to determine miRNA-like off-target silencing, the weakening of pairing stability between guide seed portion and target mRNAs optimizes the siRNA stringency ${ }^{31}$.

\section{Delivery}

The task of designing potent, highly specific and effective siRNA molecule is only the first checkpoint to cross for the development of siRNA-based therapeutics. Given that siRNA molecule has to reach the cytoplasm to fulfill its function, the tissue delivery becomes a fundamental priority to address ${ }^{106}$. After intravenous injection, siRNA starts to be systemically distributed and eliminated from the blood stream. To be recruited by the cytoplasmic RISC, the siRNA molecule has to overstep several barriers including interstitial/extravascular milieu and endolysosomal compartment ${ }^{107}$. Systemically delivered naked unmodified siRNAs, are subjected to the degradation by serum nucleases and elimination by the ReticuloEndotelial System (RES). Moreover, because of its high molecular weight and polyanionic/hydrophilic nature, siRNA molecules cannot easily diffuse across the cell membrane. Thus, the extremely short half-life and the negative physicochemical features of siRNAs have impeded the administration of non-protected siRNA-based drugs. Even though local administration has provided a valid alternative to avoid the systemic route and direct access to an interested region, is only suitable for easily accessible tissues (i.e. eye). Systemic administration remains essential for the treatment of solid tumors, but the poor pharmacokinetic profile of unformulated and unmodified siRNAs renders unfeasible their systemic delivery. In order to optimize the delivery process, carriers should comply with some rules. Prolonged blood circulation time, for example, is attained maintaining the vehicle particle size between $70-200 \mathrm{~nm}$. The obtained stealth properties essentially depend by the escape from phagocytic clearance and rapid renal elimination. Moreover, broad interactions with serum components (i.e. lipoproteins, complement proteins, albumin), which interfere with bio-availability and promote unspecific uptake by liver, spleen and RES (ReticuloEndothelial System), prevent the reaching of the target. The shielding effect of hydrophilic molecules (i.e. PolyEthylene Glycol or PEG) covering the surface of delivery vehicles, is exploited not only for minimizing the interactions with serum proteins but also provide an adequate solution to avoid the activation of the innate immune system ${ }^{108}$. The modification of the internucleotide phosphate linkage, replacing one non-bridging oxygen with one sulfur atom, alleviates the immunostimulation and improves the nuclease stability. Targeted delivery aims to address the siRNA drug toward specific tissue or cell populations with negligible toxicity for other tissues. Passive targeting takes advantage of leakiness in tumor vessels permitting carrier extravasation, whereas active targeting has the intention of limiting the delivery toward a specific target ${ }^{109}$. With the exception of hepatic delivery, passive targeting 
is not a feasible approach due to heterogeneity on tumor vascularization and tumor vessel porosity. Diverse conjugation chemistries able to link specific target molecules (i.e. antibody, peptides, aptamers, small molecules) to siRNA cargo, employ the specific ligand-receptor recognition for the uptake of certain tissue or cells ${ }^{110}$. Upon receptor-mediated endocytosis the delivery cargo is incorporated into the endosomal trafficking, final fusion of endosomes with lysosomes acidifies the lumen and liberates the nucleases promoting the degradation of siRNA. The rapid escape from endolysosomal compartment releases the siRNA into the cytosol and permits the association with RNAi machinery. Fusogenic lipids and $\mathrm{pH}$-sensitive carriers (i.e. proton escape) are some of successful strategies used for enhance the endosomal escape while protecting the siRNA from degradation ${ }^{111}$.

Of note are also self-assembled RNA superstructures, which consist of hairpin RNA structures condensed in sponge-like spherical fashion (Table 2). Thanks to the use of RNA polymerase it is possible to generate RNAi-microsponges containing about 100000 siRNA copies. The Dicer processes the hairpin RNA precursor and directly liberates active siRNA molecules into the cytosol. Furthermore, the adsorption of polyethyleneimine (PEI) onto the negatively charged RNAi-microsponge, creating a net positively charged outer layer, facilitates the cellular uptake. This self-assembled carrier yielding great siRNA loading, customized siRNA composition, stable protection from degradation, low cytotoxicity and efficient gene silencing is a promising solution to delivery ${ }^{112}$. Additional relevant means for siRNA delivery have come from the discovery of exosomes and HDL particles able to shuttle RNAs between cells ${ }^{113,114}$. Intriguing novel delivery approaches mimicking materials from endogenous sources has paved the way to the development of exosome-based vehicles (Table 2). Their endogenous derivation, impeding the recognition, the opsonization and clearance by immune system, gains crucial advantages over the other polymer-based and lipid-based carriers. Moreover, the release of the cargo directly into the cytosol, allows the bypass of the endosomal escape ${ }^{115,116}$. Additionally, starting from the nature and the structure of native $\mathrm{HDL}$, biomimetic lipoprotein carriers have been developed for efficient delivery in vivo. Reconstituted HDL-like particles (rHDL), HDL-mimicking nanoparticles are some examples of flexible, not immunological active vehicles able to transfer the siRNA cargo directly into the cytosol (Table 2$)^{117,118}$. 


\begin{tabular}{|c|c|c|}
\hline Delivery vehicles & Derivation & Advantages \\
\hline Microsponges ${ }^{106}$ & $\begin{array}{c}\text { Enzymatic } \\
\text { polymerization }\end{array}$ & $\begin{array}{c}\text { Stealth effects, customization of siRNA } \\
\text { composition, stability in biological environment, } \\
\text { high-yield siRNA loading }\end{array}$ \\
\hline Exosomes ${ }^{109,110}$ & Endogenous & $\begin{array}{c}\text { Stealth effects, release of the cargo directly into } \\
\text { the cytosol, not immunogenic }\end{array}$ \\
\hline $\begin{array}{c}\text { HDL-like } \\
\text { particles }^{111,112}\end{array}$ & Endogenous/biomimetic & $\begin{array}{l}\text { Stealth effects, bypass the Endosomal } \\
\text { compartment, immune-inert formulations }\end{array}$ \\
\hline
\end{tabular}

TABLE 2. Most recent delivery strategies

\section{CONCLUSION}

Deep understanding of RNAi pathway and nucleic acid delivery has its ultimate goal in reducing the unintended effects without compromising the silencing properties. Each step of design, synthesis and delivery of siRNA-based drugs requires adequate optimization. Rational design of siRNA molecules and tailored engineering of delivery vehicles has promoted giant progress toward clinical application. Modified siRNA provided the design of potent, highly specific and effective molecules, whereas multifunctional carriers have permitted the improvement of siRNA stability during systemic delivery and cell-specific targeting.

\section{Acknowledgements.}

The work is supported by grants of the European Commission (NMP4-LA-2011-262943, MULTIFUN), the Spanish Ministry of Economy (CTQ2014-52588-R, RTC-2014-2038-1), Generalitat de Catalunya (2014/SGR/624) and the Instituto de Salud Carlos III (CB06_01_0019) with assistance from the European Regional Development Fund.

\section{REFERENCES}

1. Napoli C, Lemieux C, Jorgensen R. Introduction of a Chimeric Chalcone Synthase Gene into Petunia Results in Reversible Co-Suppression of Homologous Genes in trans. Plant Cell 1990, 2:279-289.

2. Fire A, Xu S, Montgomery MK, Kostas SA, Driver SE, Mello CC. Potent and specific genetic interference by double-stranded RNA in Caenorhabditis elegans. Nature 1998, 391:806-811.

3. Hammond SM, Bernstein E, Beach D, Hannon GJ. An RNA-directed nuclease mediates post-transcriptional gene silencing in Drosophila cells. Nature 2000, 404:293-296. 
4. Liu J, Carmell MA, Rivas FV, Marsden CG, Thomson JM, Song JJ, Hammond SM, JoshuaTor L, Hannon GJ. Argonaute2 is the catalytic engine of mammalian RNAi. Science 2004, 305:1437-1441.

5. Hamilton AJ, Baulcombe DC. A species of small antisense RNA in posttranscriptional gene silencing in plants. Science 1999, 286:950-952.

6. Elbashir SM, Harborth J, Lendeckel W, Yalcin A, Weber K, Tuschl T. Duplexes of 21nucleotide RNAs mediate RNA interference in cultured mammalian cells. Nature 2001, 411:494-498.

7. Jackson AL, Bartz SR, Schelter J, Kobayashi SV, Burchard J, Mao M, Li B, Cavet G, Linsley PS. Expression profiling reveals off-target gene regulation by RNAi. Nat Biotechnol 2003, 21:635-637.

8. Chang Cl, Kim HA, Dua P, Kim S, Li CJ, Lee DK. Structural diversity repertoire of gene silencing small interfering RNAs. Nucleic Acid Ther 2011, 21:125-131.

9. Tafer H. Bioinformatics of siRNA design. Methods Mol Biol 2014, 1097:477-490.

10. Tafer H, Ameres SL, Obernosterer G, Gebeshuber CA, Schroeder R, Martinez J, Hofacker IL. The impact of target site accessibility on the design of effective siRNAs. Nat Biotechnol 2008, 26:578-583.

11. Ameres SL, Martinez J, Schroeder R. Molecular basis for target RNA recognition and cleavage by human RISC. Cell 2007, 130:101-112.

12. Deleavey GF, Damha MJ. Designing chemically modified oligonucleotides for targeted gene silencing. Chem Biol 2012, 19:937-954.

13. Grijalvo S, Avino A, Eritja R. Oligonucleotide delivery: a patent review (2010 - 2013). Expert Opin Ther Pat 2014, 24:801-819.

14. Meister G. Argonaute proteins: functional insights and emerging roles. Nat Rev Genet 2013, 14:447-459.

15. Frank F, Sonenberg N, Nagar B. Structural basis for 5'-nucleotide base-specific recognition of guide RNA by human AGO2. Nature 2010, 465:818-822.

16. Wang HW, Noland C, Siridechadilok B, Taylor DW, Ma E, Felderer K, Doudna JA, Nogales E. Structural insights into RNA processing by the human RISC-loading complex. Nat Struct Mol Biol 2009, 16:1148-1153.

17. Song JJ, Liu J, Tolia NH, Schneiderman J, Smith SK, Martienssen RA, Hannon GJ, JoshuaTor L. The crystal structure of the Argonaute2 PAZ domain reveals an RNA binding motif in RNAi effector complexes. Nat Struct Biol 2003, 10:1026-1032.

18. Kwak PB, Tomari $\mathrm{Y}$. The $\mathrm{N}$ domain of Argonaute drives duplex unwinding during RISC assembly. Nat Struct Mol Biol 2012, 19:145-151.

19. Jinek M, Doudna JA. A three-dimensional view of the molecular machinery of RNA interference. Nature 2009, 457:405-412.

20. Rana TM. Illuminating the silence: understanding the structure and function of small RNAs. Nat Rev Mol Cell Biol 2007, 8:23-36.

21. Wee LM, Flores-Jasso CF, Salomon WE, Zamore PD. Argonaute divides its RNA guide into domains with distinct functions and RNA-binding properties. Cell 2012, 151:10551067.

22. Schirle NT, Sheu-Gruttadauria J, MacRae IJ. Structural basis for microRNA targeting. Science 2014, 346:608-613.

23. Lima WF, Wu H, Nichols JG, Sun H, Murray HM, Crooke ST. Binding and cleavage specificities of human Argonaute2. J Biol Chem 2009, 284:26017-26028.

24. Felice KM, Salzman DW, Shubert-Coleman J, Jensen KP, Furneaux HM. The 5' terminal uracil of let-7a is critical for the recruitment of mRNA to Argonaute2. Biochem J 2009, 422:329-341.

25. Boland A, Tritschler F, Heimstadt S, Izaurralde E, Weichenrieder O. Crystal structure and ligand binding of the MID domain of a eukaryotic Argonaute protein. EMBO Rep 2010, 11:522-527. 
26. Schirle NT, Sheu-Gruttadauria J, Chandradoss SD, Joo C, MacRae IJ. Water-mediated recognition of t1-adenosine anchors Argonaute2 to microRNA targets. Elife 2015, 4.

27. Onizuka K, Harrison JG, Ball-Jones AA, Ibarra-Soza JM, Zheng Y, Ly D, Lam W, Mac S, Tantillo DJ, Beal PA. Short interfering RNA guide strand modifiers from computational screening. J Am Chem Soc 2013, 135:17069-17077.

28. Wang Y, Juranek S, Li H, Sheng G, Wardle GS, Tuschl T, Patel DJ. Nucleation, propagation and cleavage of target RNAs in Ago silencing complexes. Nature 2009, 461:754-761.

29. Wang X. Composition of seed sequence is a major determinant of microRNA targeting patterns. Bioinformatics 2014, 30:1377-1383.

30. Jackson AL, Burchard J, Schelter J, Chau BN, Cleary M, Lim L, Linsley PS. Widespread siRNA "off-target" transcript silencing mediated by seed region sequence complementarity. RNA 2006, 12:1179-1187.

31. Ui-Tei K. Optimal choice of functional and off-target effect-reduced siRNAs for RNAi therapeutics. Front Genet 2013, 4:107.

32. Ui-Tei K, Naito Y, Nishi K, Juni A, Saigo K. Thermodynamic stability and Watson-Crick base pairing in the seed duplex are major determinants of the efficiency of the siRNAbased off-target effect. Nucleic Acids Res 2008, 36:7100-7109.

33. Bramsen JB, Pakula MM, Hansen TB, Bus C, Langkjaer N, Odadzic D, Smicius R, Wengel SL, Chattopadhyaya J, Engels JW, et al. A screen of chemical modifications identifies position-specific modification by UNA to most potently reduce siRNA off-target effects. Nucleic Acids Res 2010, 38:5761-5773.

34. Gu S, Zhang $Y$, Jin L, Huang $Y$, Zhang F, Bassik MC, Kampmann M, Kay MA. Weak base pairing in both seed and $3^{\prime}$ regions reduces RNAi off-targets and enhances si/shRNA designs. Nucleic Acids Res 2014, 42:12169-12176.

35. Vaish N, Chen F, Seth S, Fosnaugh K, Liu Y, Adami R, Brown T, Chen Y, Harvie P, Johns R, et al. Improved specificity of gene silencing by siRNAs containing unlocked nucleobase analogs. Nucleic Acids Res 2011, 39:1823-1832.

36. Jackson AL, Burchard J, Leake D, Reynolds A, Schelter J, Guo J, Johnson JM, Lim L, Karpilow J, Nichols K, et al. Position-specific chemical modification of siRNAs reduces "off-target" transcript silencing. RNA 2006, 12:1197-1205.

37. Ui-Tei K, Naito Y, Zenno S, Nishi K, Yamato K, Takahashi F, Juni A, Saigo K. Functional dissection of siRNA sequence by systematic DNA substitution: modified siRNA with a DNA seed arm is a powerful tool for mammalian gene silencing with significantly reduced off-target effect. Nucleic Acids Res 2008, 36:2136-2151.

38. Schirle NT, MacRae IJ. The crystal structure of human Argonaute2. Science 2012, 336:1037-1040.

39. Kume H, Hino K, Galipon J, Ui-Tei K. A-to-I editing in the miRNA seed region regulates target mRNA selection and silencing efficiency. Nucleic Acids Res 2014, 42:1005010060.

40. Sipa K, Sochacka E, Kazmierczak-Baranska J, Maszewska M, Janicka M, Nowak G, Nawrot B. Effect of base modifications on structure, thermodynamic stability, and gene silencing activity of short interfering RNA. RNA 2007, 13:1301-1316.

41. Chak LL, Okamura K. Argonaute-dependent small RNAs derived from single-stranded, non-structured precursors. Front Genet 2014, 5:172.

42. Leuschner PJ, Ameres SL, Kueng S, Martinez J. Cleavage of the siRNA passenger strand during RISC assembly in human cells. EMBO Rep 2006, 7:314-320.

43. Gu S, Jin L, Huang Y, Zhang F, Kay MA. Slicing-independent RISC activation requires the argonaute PAZ domain. Curr Biol 2012, 22:1536-1542.

44. Takahashi T, Zenno S, Ishibashi O, Takizawa T, Saigo K, Ui-Tei K. Interactions between the non-seed region of siRNA and RNA-binding RLC/RISC proteins, Ago and TRBP, in mammalian cells. Nucleic Acids Res 2014, 42:5256-5269. 
45. Zheng J, Zhang L, Zhang J, Wang X, Ye K, Xi Z, Du Q, Liang Z. Single modification at position 14 of siRNA strand abolishes its gene-silencing activity by decreasing both RISC loading and target degradation. FASEB J 2013, 27:4017-4026.

46. Prakash TP, Allerson CR, Dande P, Vickers TA, Sioufi N, Jarres R, Baker BF, Swayze EE, Griffey $\mathrm{RH}$, Bhat B. Positional effect of chemical modifications on short interference RNA activity in mammalian cells. J Med Chem 2005, 48:4247-4253.

47. Alagia A, Terrazas M, Eritja R. Modulation of the RNA Interference Activity Using Central Mismatched siRNAs and Acyclic Threoninol Nucleic Acids (aTNA) Units. Molecules 2015, 20:7602-7619.

48. Elbashir SM, Martinez J, Patkaniowska A, Lendeckel W, Tuschl T. Functional anatomy of siRNAs for mediating efficient RNAi in Drosophila melanogaster embryo lysate. EMBO J 2001, 20:6877-6888.

49. Deerberg A, Willkomm S, Restle T. Minimal mechanistic model of siRNA-dependent target RNA slicing by recombinant human Argonaute 2 protein. Proc Natl Acad Sci U S A 2013, 110:17850-17855.

50. Zander A, Holzmeister P, Klose D, Tinnefeld P, Grohmann D. Single-molecule FRET supports the two-state model of Argonaute action. RNA Biol 2014, 11:45-56.

51. Alagia A, Terrazas M, Eritja R. RNA/aTNA chimeras: RNAi effects and nucleases resistance of single and double stranded RNAs. Molecules 2014, 19:17872-17896.

52. Terrazas M, Ocampo SM, Perales JC, Marquez VE, Eritja R. Effect of north bicyclo[3.1.0] hexane 2'-deoxy-pseudosugars on RNA interference: a novel class of siRNA modification. Chembiochem 2011, 12:1056-1065.

53. Potenza N, Moggio L, Milano G, Salvatore V, Di Blasio B, Russo A, Messere A. RNA interference in mammalia cells by RNA-3'-PNA chimeras. Int J Mol Sci 2008, 9:299-315.

54. Kamiya Y, Takai J, Ito H, Murayama K, Kashida H, Asanuma H. Enhancement of stability and activity of siRNA by terminal substitution with serinol nucleic acid (SNA). Chembiochem 2014, 15:2549-2555.

55. Terrazas M, Alagia A, Faustino I, Orozco M, Eritja R. Functionalization of the 3 '-ends of DNA and RNA strands with $\mathrm{N}$-ethyl-N-coupled nucleosides: a promising approach to avoid 3'-exonuclease-catalyzed hydrolysis of therapeutic oligonucleotides. Chembiochem 2013, 14:510-520.

56. Gaglione M, Potenza N, Di Fabio G, Romanucci V, Mosca N, Russo A, Novellino E, Cosconati $S$, Messere A. Tuning RNA Interference by Enhancing siRNA/PAZ Recognition. ACS Med Chem Lett 2013, 4:75-78.

57. Somoza A, Terrazas M, Eritja R. Modified siRNAs for the study of the PAZ domain. Chem Commun (Camb) 2010, 46:4270-4272.

58. Kandeel M, Kitade $\mathrm{Y}$. In silico molecular docking analysis of the human Argonaute 2 PAZ domain reveals insights into RNA interference. J Comput Aided Mol Des 2013, 27:605-614.

59. Zhou J, Song MS, Jacobi AM, Behlke MA, Wu X, Rossi JJ. Deep Sequencing Analyses of DsiRNAs Reveal the Influence of 3' Terminal Overhangs on Dicing Polarity, Strand Selectivity, and RNA Editing of siRNAs. Mol Ther Nucleic Acids 2012, 1:e17.

60. Xu L, Wang X, He H, Zhou J, Li X, Ma H, Li Z, Zeng Y, Shao R, Cen S, et al. Structurebased design of novel chemical modification of the 3 '-overhang for optimization of short interfering RNA performance. Biochemistry 2015, 54:1268-1277.

61. Sano M, Sierant M, Miyagishi M, Nakanishi M, Takagi Y, Sutou S. Effect of asymmetric terminal structures of short RNA duplexes on the RNA interference activity and strand selection. Nucleic Acids Res 2008, 36:5812-5821.

62. Hoerter JA, Krishnan V, Lionberger TA, Walter NG. siRNA-like double-stranded RNAs are specifically protected against degradation in human cell extract. PLoS One 2011, 6:e20359. 
63. Bartlett DW, Davis ME. Insights into the kinetics of siRNA-mediated gene silencing from live-cell and live-animal bioluminescent imaging. Nucleic Acids Res 2006, 34:322333.

64. Bartlett DW, Davis ME. Effect of siRNA nuclease stability on the in vitro and in vivo kinetics of siRNA-mediated gene silencing. Biotechnol Bioeng 2007, 97:909-921.

65. Cuccato G, Polynikis A, Siciliano V, Graziano M, di Bernardo M, di Bernardo D. Modeling RNA interference in mammalian cells. BMC Syst Biol 2011, 5:19.

66. Bramsen JB, Kjems J. Development of Therapeutic-Grade Small Interfering RNAs by Chemical Engineering. Front Genet 2012, 3:154.

67. Snead NM, Wu X, Li A, Cui Q, Sakurai K, Burnett JC, Rossi JJ. Molecular basis for improved gene silencing by Dicer substrate interfering RNA compared with other siRNA variants. Nucleic Acids Res 2013, 41:6209-6221.

68. Abe $N$, Abe $H$, Nagai $C$, Harada $M$, Hatakeyama $H$, Harashima $H$, Ohshiro T, Nishihara $M$, Furukawa $K$, Maeda $M$, et al. Synthesis, structure, and biological activity of dumbbell-shaped nanocircular RNAs for RNA interference. Bioconjug Chem 2011, 22:2082-2092.

69. Wei JX, Yang J, Sun JF, Jia LT, Zhang Y, Zhang HZ, Li X, Meng YL, Yao LB, Yang AG. Both strands of siRNA have potential to guide posttranscriptional gene silencing in mammalian cells. PLoS One 2009, 4:e5382.

70. Schwarz DS, Hutvagner G, Haley B, Zamore PD. Evidence that siRNAs function as guides, not primers, in the Drosophila and human RNAi pathways. Mol Cell 2002, 10:537-548.

71. Kenski DM, Cooper AJ, Li JJ, Willingham AT, Haringsma HJ, Young TA, Kuklin NA, Jones $\mathrm{JJ}$, Cancilla MT, McMasters DR, et al. Analysis of acyclic nucleoside modifications in siRNAs finds sensitivity at position 1 that is restored by 5 '-terminal phosphorylation both in vitro and in vivo. Nucleic Acids Res 2010, 38:660-671.

72. Chen PY, Weinmann L, Gaidatzis D, Pei Y, Zavolan M, Tuschl T, Meister G. Strandspecific 5'-O-methylation of siRNA duplexes controls guide strand selection and targeting specificity. RNA 2008, 14:263-274.

73. Snead NM, Escamilla-Powers JR, Rossi JJ, McCaffrey AP. 5' Unlocked Nucleic Acid Modification Improves siRNA Targeting. Mol Ther Nucleic Acids 2013, 2:e103.

74. Bramsen JB, Laursen MB, Damgaard CK, Lena SW, Babu BR, Wengel J, Kjems J. Improved silencing properties using small internally segmented interfering RNAs. Nucleic Acids Res 2007, 35:5886-5897.

75. Chang $\mathrm{Cl}$, Yoo JW, Hong SW, Lee SE, Kang HS, Sun X, Rogoff HA, Ban C, Kim S, Li CJ, et al. Asymmetric shorter-duplex siRNA structures trigger efficient gene silencing with reduced nonspecific effects. Mol Ther 2009, 17:725-732.

76. Sun X, Rogoff HA, Li CJ. Asymmetric RNA duplexes mediate RNA interference in mammalian cells. Nat Biotechnol 2008, 26:1379-1382.

77. Yuan Z, Wu X, Liu C, Xu G, Wu Z. Asymmetric siRNA: new strategy to improve specificity and reduce off-target gene expression. Hum Gene Ther 2012, 23:521-532.

78. Lima WF, Prakash TP, Murray HM, Kinberger GA, Li W, Chappell AE, Li CS, Murray SF, Gaus H, Seth PP, et al. Single-stranded siRNAs activate RNAi in animals. Cell 2012, 150:883-894.

79. Kenski DM, Willingham AT, Haringsma HJ, Li JJ, Flanagan WM. In vivo activity and duration of short interfering RNAs containing a synthetic 5'-phosphate. Nucleic Acid Ther 2012, 22:90-95.

80. Tiemann K, Hohn B, Ehsani A, Forman SJ, Rossi JJ, Saetrom P. Dual-targeting siRNAs. RNA 2010, 16:1275-1284.

81. Hossbach M, Gruber J, Osborn M, Weber K, Tuschl T. Gene silencing with siRNA duplexes composed of target-mRNA-complementary and partially palindromic or partially complementary single-stranded siRNAs. RNA Biol 2006, 3:82-89. 
82. Saetrom P. Designing dual-targeting siRNA duplexes having two active strands that combine siRNA and microRNA-like targeting. Methods Mol Biol 2013, 942:169-177.

83. Kawai T, Akira S. The roles of TLRs, RLRs and NLRs in pathogen recognition. Int Immunol 2009, 21:317-337.

84. Robbins M, Judge A, MacLachlan I. siRNA and innate immunity. Oligonucleotides 2009, 19:89-102.

85. Jackson AL, Linsley PS. Recognizing and avoiding siRNA off-target effects for target identification and therapeutic application. Nat Rev Drug Discov 2010, 9:57-67.

86. Gantier MP, Tong S, Behlke MA, Irving AT, Lappas M, Nilsson UW, Latz E, McMillan NA, Williams BR. Rational design of immunostimulatory siRNAs. Mol Ther 2010, 18:785795.

87. Sadler AJ, Williams BR. Interferon-inducible antiviral effectors. Nat Rev Immunol 2008, 8:559-568.

88. Gantier MP, Williams BR. The response of mammalian cells to double-stranded RNA. Cytokine Growth Factor Rev 2007, 18:363-371.

89. Uzri D, Gehrke L. Nucleotide sequences and modifications that determine RIG-I/RNA binding and signaling activities. J Virol 2009, 83:4174-4184.

90. Eberle F, Giessler K, Deck C, Heeg K, Peter M, Richert C, Dalpke AH. Modifications in small interfering RNA that separate immunostimulation from RNA interference. $J$ Immunol 2008, 180:3229-3237.

91. Peacock H, Fucini RV, Jayalath $P$, Ibarra-Soza JM, Haringsma HJ, Flanagan WM, Willingham A, Beal PA. Nucleobase and ribose modifications control immunostimulation by a microRNA-122-mimetic RNA. J Am Chem Soc 2011, 133:92009203.

92. Fucini RV, Haringsma HJ, Deng $P$, Flanagan WM, Willingham AT. Adenosine modification may be preferred for reducing siRNA immune stimulation. Nucleic Acid Ther 2012, 22:205-210.

93. Valenzuela RA, Suter SR, Ball-Jones AA, Ibarra-Soza JM, Zheng Y, Beal PA. Base modification strategies to modulate immune stimulation by an siRNA. Chembiochem 2015, 16:262-267.

94. Poeck H, Besch R, Maihoefer C, Renn M, Tormo D, Morskaya SS, Kirschnek S, Gaffal E, Landsberg J, Hellmuth J, et al. 5'-Triphosphate-siRNA: turning gene silencing and Rig-I activation against melanoma. Nat Med 2008, 14:1256-1263.

95. Ebert G, Poeck H, Lucifora J, Baschuk N, Esser K, Esposito I, Hartmann G, Protzer U. 5' Triphosphorylated small interfering RNAs control replication of hepatitis B virus and induce an interferon response in human liver cells and mice. Gastroenterology 2011, 141:696-706, 706 e691-693.

96. Han Q, Zhang C, Zhang J, Tian Z. Reversal of hepatitis B virus-induced immune tolerance by an immunostimulatory $3 p-H B x-s i R N A s$ in a retinoic acid inducible gene Idependent manner. Hepatology 2011, 54:1179-1189.

97. Chevalier C, Saulnier A, Benureau Y, Flechet D, Delgrange D, Colbere-Garapin F, Wychowski C, Martin A. Inhibition of hepatitis $C$ virus infection in cell culture by small interfering RNAs. Mol Ther 2007, 15:1452-1462.

98. Kortylewski M, Swiderski P, Herrmann A, Wang L, Kowolik C, Kujawski M, Lee H, Scuto $A$, Liu $Y$, Yang $C$, et al. In vivo delivery of siRNA to immune cells by conjugation to a TLR9 agonist enhances antitumor immune responses. Nat Biotechnol 2009, 27:925932.

99. Khairuddin N, Gantier MP, Blake SJ, Wu SY, Behlke MA, Williams BR, McMillan NA. siRNA-induced immunostimulation through TLR7 promotes antitumoral activity against HPV-driven tumors in vivo. Immunol Cell Biol 2012, 90:187-196. 
100. Meng G, Xia M, Xu C, Yuan D, Schnurr M, Wei J. Multifunctional antitumor molecule 5'triphosphate siRNA combining glutaminase silencing and RIG-I activation. Int J Cancer 2014, 134:1958-1971.

101. Ellermeier J, Wei J, Duewell P, Hoves S, Stieg MR, Adunka T, Noerenberg D, Anders HJ, Mayr D, Poeck $\mathrm{H}$, et al. Therapeutic efficacy of bifunctional siRNA combining TGF-beta1 silencing with RIG-I activation in pancreatic cancer. Cancer Res 2013, 73:1709-1720.

102. Schnurr M, Duewell P. Breaking tumor-induced immunosuppression with 5'triphosphate siRNA silencing TGFbeta and activating RIG-I. Oncoimmunology 2013, 2:e24170.

103. Anderson E, Boese $Q$, Khvorova A, Karpilow J. Identifying siRNA-induced off-targets by microarray analysis. Methods Mol Biol 2008, 442:45-63.

104. Dua P, Yoo JW, Kim S, Lee DK. Modified siRNA structure with a single nucleotide bulge overcomes conventional siRNA-mediated off-target silencing. Mol Ther 2011, 19:16761687.

105. Anderson EM, Birmingham A, Baskerville S, Reynolds A, Maksimova E, Leake D, Fedorov $Y$, Karpilow J, Khvorova A. Experimental validation of the importance of seed complement frequency to siRNA specificity. RNA 2008, 14:853-861.

106. Whitehead KA, Langer R, Anderson DG. Knocking down barriers: advances in siRNA delivery. Nat Rev Drug Discov 2009, 8:129-138.

107. Tiwari G, Tiwari R, Sriwastawa B, Bhati L, Pandey S, Pandey P, Bannerjee SK. Drug delivery systems: An updated review. Int J Pharm Investig 2012, 2:2-11.

108. Kumar V, Qin J, Jiang Y, Duncan RG, Brigham B, Fishman S, Nair JK, Akinc A, Barros SA, Kasperkovitz PV. Shielding of Lipid Nanoparticles for siRNA Delivery: Impact on Physicochemical Properties, Cytokine Induction, and Efficacy. Mol Ther Nucleic Acids 2014, 3:e210.

109. Shen H, Sun T, Ferrari M. Nanovector delivery of siRNA for cancer therapy. Cancer Gene Ther 2012, 19:367-373.

110. Nielsen C, Kjems J, Sorensen KR, Engelholm LH, Behrendt N. Advances in targeted delivery of small interfering RNA using simple bioconjugates. Expert Opin Drug Deliv 2014, 11:791-822.

111. Shete HK, Prabhu RH, Patravale VB. Endosomal escape: a bottleneck in intracellular delivery. J Nanosci Nanotechnol 2014, 14:460-474.

112. Lee JB, Hong J, Bonner DK, Poon Z, Hammond PT. Self-assembled RNA interference microsponges for efficient siRNA delivery. Nat Mater 2012, 11:316-322.

113. Valadi H, Ekstrom K, Bossios A, Sjostrand M, Lee JJ, Lotvall JO. Exosome-mediated transfer of mRNAs and microRNAs is a novel mechanism of genetic exchange between cells. Nat Cell Biol 2007, 9:654-659.

114. Vickers KC, Palmisano BT, Shoucri BM, Shamburek RD, Remaley AT. MicroRNAs are transported in plasma and delivered to recipient cells by high-density lipoproteins. Nat Cell Biol 2011, 13:423-433.

115. van den Boorn JG, Dassler J, Coch C, Schlee M, Hartmann G. Exosomes as nucleic acid nanocarriers. Adv Drug Deliv Rev 2013, 65:331-335.

116. Kooijmans SA, Vader P, van Dommelen SM, van Solinge WW, Schiffelers RM. Exosome mimetics: a novel class of drug delivery systems. Int J Nanomedicine 2012, 7:15251541.

117. Ding Y, Wang Y, Zhou J, Gu X, Wang W, Liu C, Bao X, Wang C, Li Y, Zhang Q. Direct cytosolic siRNA delivery by reconstituted high density lipoprotein for target-specific therapy of tumor angiogenesis. Biomaterials 2014, 35:7214-7227.

118. Nakayama T, Butler JS, Sehgal A, Severgnini M, Racie T, Sharman J, Ding F, Morskaya SS, Brodsky J, Tchangov L, et al. Harnessing a physiologic mechanism for siRNA delivery with mimetic lipoprotein particles. Mol Ther 2012, 20:1582-1589. 


\section{Further Reading}

Howard KA. RNA interference from biology to therapeutics. Springer. US. 2013. 340.

Taxman, DJ. siRNA design: Methods and Protocols. Humana Press. US. 2012. 392.

Cheng K, Mahato RI. Advanced delivery and therapeutic applications of RNAi. Wiley. US. 2013. 543.

Meares CF, Yokoyama M. Special issue on gene silencing and delivery. Acc Chem Res 2012, 45:959-1171.

Burnett JC, Rossi JJ. RNA-based therapeutics: current progress and future prospects. Chem Biol 2012, 19:60-71.

Chakraborty C. Potentiality of small interfering RNAs (siRNA) as recent therapeutic targets for gene-silencing. Curr Drug Targets 2007, 8:469-482.

Siomi H, Siomi MC. On the road to reading the RNA-interference code. Nature 2009, 457:396404.

Shukla S, Sumaria CS, Pradeepkumar PI. Exploring chemical modifications for siRNA therapeutics: a structural and functional outlook. ChemMedChem 2010, 5:328-349.

Stanton MG, Colletti SL. Medicinal chemistry of siRNA delivery. J. Med. Chem. 2010, 53:78877901.

\section{Cross References}

1. Snead NM, Rossi JJ. Biogenenis and function of endogenous and exogeneous siRNAs. WIRES RNA 2010, 1: 117-131.

2. Ford LP, Toloue MM. Delivery of RNAi mediators. WIREs RNA 2010, 1:341-350.

3. Mohr SE, Perrimon N. RNAi screening: new approaches, understandings, and organism. WIREs RNA 2012, 3:145-158. 